\title{
How to encourage trial reporting
}

\author{
Reporting of data from clinical trials comes slowly or not at all. Impending regulations in the US promise to improve \\ the situation, but full compliance will require better incentives from institutions and a greater understanding that \\ reporting data does not jeopardize the publication of results.
}

$\mathrm{E}$ nhancing data sharing is among the goals set by the 'Cancer Moonshot' initiative, which the White House announced earlier this year. At the Cancer Moonshot Summit held on 29 June at Howard University, US Vice President Joe Biden took this one step further when he floated the idea that the government could withdraw federal funds from US institutions that fail to report clinical trial results. Biden cited a recent investigation by STAT that exposed widespread noncompliance with current clinical trial data-sharing policies. Currently, the US Food and Drug Administration (FDA) Amendments Act 2007 (FDAAA) requires US clinical trials evaluating drugs or devices that have been approved by the FDA to publish a summary of their results on the ClinicalTrials.gov website within one year of trial completion or termination, with the exception of phase 1 trials, which are not subject to this obligation.

The STAT investigation and other analyses indicate that more than $80 \%$ of clinical trials violate this law by going unreported (N. Engl. J. Med. 372, 1031-1039, 2015; BMJ 352, i637, 2016). The problem is particularly acute at academic medical institutions, which lag behind biopharmaceutical companies in their rates of trial reporting. In response, institutions such as the Memorial Sloan Kettering Cancer Center in New York City have expressed intentions to improve their reporting. However, timely dissemination of clinical trial results will require more than good intentions. For example, institutional review boards at academic centers could establish internal policies to sanction or incentivize compliance by investigators for the trials approved by these boards.

The facilitation of access to clinical trial results can minimize the duplication of studies, thereby optimizing the allocation of resources such as money and human labor. Transparent reporting of such data also provides physicians with the knowledge that they need to make informed treatment decisions. The absence of this knowledge might result in unnecessary exposure of patients to unsafe or ineffective therapies. Finally, dissemination of the outcomes of trials is ethical because of the involvement of human subjects in such research, and furthermore, it could help study participants to gain greater awareness of the value of their commitment and involvement in research. It is no surprise, then, that efforts in favor of clinical trial data reporting are taking place beyond the US. Two years ago, the EU emphasized that it requires data reporting from investigators. Last year, the World Health Organization also called for more robust clinical trial data reporting.

In the US, the FDAAA stipulates that institutions that fail to comply can incur penalties of up to $\$ 10,000$ for each day's delay in delivering the required summaries. However, the STAT report revealed that, despite the widespread lack of adherence to the law, no fines have been levied. This lack of enforcement sends the wrong message, effectively implying that institutions may sidestep the law with impunity.

A proposed change by the US Department of Health and Human Services would tighten up existing rules for the submission of data to ClinicalTrials.gov by requiring data to be reported regardless of whether the treatment has been approved by the FDA. Unfortunately, investigators from industry and academic institutions worry that the publication of clinical trial result summaries in public repositories might jeopardize the interest of scientific journals in subsequently publishing papers that present these results. However, earlier this year, the International Committee of Medical Journal Editors declared that editors of their journals "will not consider the deposition of data in a registry to constitute prior publication" (N. Engl. J. Med. 374, 384-386, 2016). Nature Medicine adheres to this same principle. Another complaint voiced by investigators is that peer-reviewed journals are interested in publishing positive, but not negative, clinical trial results. But the publication of results in peer-reviewed journals is independent from reporting them on clinical trial registries, and thus biased publication preferences are not a justification for failure to deposit clinical trial data in designated public repositories.

A second potential change, proposed by the US National Institutes of Health $(\mathrm{NIH})$, will require all clinical trials funded by the NIH to report data-including phase 1 data-regardless of whether they are subject to the FDAAA. But non-NIH-funded clinical trials are exempt from these policies, and so this new legislation is not a cure-all. The inclusion of phase 1 data under the FDAAA rules for required reporting, even when these early trials are not funded by the NIH, would give a fuller picture of the progress being made in medical research.

Regulatory agencies have the duty and the responsibility to fully report clinical trial results and enhance the transparency of clinical research. Institutions and industry should get onboard and both offer incentives and shift the mentality of investigators to overcome hesitation to share trial results. The end goal could not be more worthy. 